\title{
New existence criteria for periodic solution to a Duffing $p$-Laplacian-Like equation
}

\author{
Zhiyan $\mathrm{Li}^{1, \mathrm{a}}$ \\ ${ }^{1}$ Department of Mathematics and Physics, Hohai University, Changzhou Campus, \\ Changzhou, P.R. of China \\ alizhiyan_hh@163.com
}

Keywords: $\boldsymbol{P}$-Laplacian-Like operator, Periodic solution, Degree theory, Duffing equation

\begin{abstract}
In this study, we investigate a kind of Duffing type ${ }^{p}$-Laplacian-Like equation. Some new criteria for guaranteeing the existence and uniqueness of periodic solution of this equation are given by using the Manásevich-Mawhin continuation theorem and some analysis techniques. Our results improve and extend some known results from the literature.
\end{abstract}

\section{Introduction}

In this paper, we consider the existence and uniqueness of periodic solution for the following nonlinear equation with p-Laplacian-Like operator

$\left(\phi\left(x^{\prime}(t)\right)\right)^{\prime}+C x^{\prime}(t)+g(t, x(t))=e(t)$,

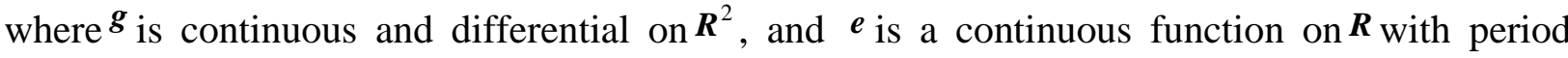
$\boldsymbol{T}>0, \boldsymbol{C}$ is a given constant; moreover, $\phi$ is called $\boldsymbol{p}$-Laplacian-Like operator satisfying the following conditions:

$\left(\mathrm{H}_{1}\right) \forall \boldsymbol{x}_{1}, \boldsymbol{x}_{2} \in \boldsymbol{R}, \boldsymbol{x}_{1} \neq \boldsymbol{x}_{2},\left[\phi\left(\boldsymbol{x}_{1}\right)-\phi\left(\boldsymbol{x}_{2}\right)\right] \cdot\left(\boldsymbol{x}_{1}-\boldsymbol{x}_{2}\right)>0, \phi$ is continuous and $\phi(0)=0$;

$\left(\mathrm{H}_{2}\right)$ There exists a function $\alpha:[0,+\infty) \rightarrow[0,+\infty)$ such that $\lim _{s \rightarrow+\infty} \alpha(s)=+\infty$ and

$$
\phi(\boldsymbol{x}) \cdot \boldsymbol{x} \geq \alpha(|\boldsymbol{x}|)|\boldsymbol{x}|^{2}, \quad \forall \boldsymbol{x} \in \boldsymbol{R} .
$$

It's obvious that under conditions $\left(\mathrm{H}_{1}\right)$ and $\left(\mathrm{H}_{2}\right), \phi$ is an isomorphism from $\boldsymbol{R}$ onto $\boldsymbol{R}$ and is increasing on $\boldsymbol{R}$. As is known, p-Laplacian operator $\varphi_{p}(s)=|s|^{p-2} s, p>1$, it is a special case of $\phi$. However, there are few results about the existence of periodic solutions to Eq. (1), the difficulty lies in that $\phi$ is more complicated than p-Laplacian operator and $\phi$ has no concrete form. There are many results about p-Laplacian operator, see examples [1-7].

Recently, Wang [8] considered the following p-Laplacian equation

$\left(\varphi_{p}\left(x^{\prime}(t)\right)\right)^{\prime}+C x^{\prime}(t)+g(t, x(t))=e(t)$.

Under some conditions, they have obtained the existence and uniqueness.

Remark. When $\phi=\varphi_{p}(s)$, Eq. (1) is Eq. (2), that is to say Eq. (2) is a special case of Eq. (1). Our main results read as follows:

Theorem 1. In the problem (1), assume that

$\left(\mathrm{A}_{1}\right)$ There exists a constant $\boldsymbol{d}>0$ such that $\boldsymbol{x} \boldsymbol{g}(\boldsymbol{t}, \boldsymbol{x})<0, \forall \boldsymbol{t} \in \boldsymbol{R}$ and $|\boldsymbol{x}| \geq \boldsymbol{d}$,

$\left(\mathrm{A}_{2}\right)\left[\boldsymbol{g}\left(\boldsymbol{t}, \boldsymbol{u}_{1}\right)-\boldsymbol{g}\left(\boldsymbol{t}, \boldsymbol{u}_{2}\right)\right] \cdot\left(\boldsymbol{u}_{1}-\boldsymbol{u}_{2}\right)<0, \boldsymbol{u}_{1} \neq \boldsymbol{u}_{2}$ hold.Then Eq. (1) has an unique periodic solution.

Some lemmas and notationsThe following lemma 1 is necessary for the proof of Theorem1.

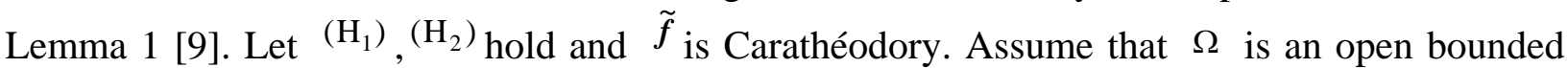
set in $C_{T}^{1}$ such that the following conditions are satisfied:

$\left(\mathrm{S}_{1}\right)$ For each $\lambda \in(0,1)$, the problem $\left(\phi\left(\boldsymbol{x}^{\prime}(\boldsymbol{t})\right)\right)^{\prime}=\lambda \tilde{f}\left(\boldsymbol{t}, \boldsymbol{x}, \boldsymbol{x}^{\prime}\right), \boldsymbol{x}(0)=\boldsymbol{x}(\boldsymbol{T}), \boldsymbol{x}^{\prime}(0)=\boldsymbol{x}^{\prime}(\boldsymbol{T})$ has no solution on $\partial \Omega$, 
$\left(\mathrm{S}_{2}\right)$ The equation $\boldsymbol{F}(\boldsymbol{a})=\frac{1}{\boldsymbol{T}} \int_{0}^{T} \tilde{\boldsymbol{f}}(\boldsymbol{t}, \boldsymbol{a}, 0) \boldsymbol{d} \boldsymbol{t}=0$ has no solution on $\partial \Omega \cap \boldsymbol{R}$.

$\left(\mathrm{S}_{3}\right)$ The Brower degree $\operatorname{deg}(\boldsymbol{F}, \Omega \cap \boldsymbol{R}, 0) \neq 0$. Then the periodic boundary value problem $\left(\phi\left(\boldsymbol{x}^{\prime}(\boldsymbol{t})\right)\right)^{\prime}=\tilde{\boldsymbol{f}}\left(\boldsymbol{t}, \boldsymbol{x}, \boldsymbol{x}^{\prime}\right), \boldsymbol{x}(0)=\boldsymbol{x}(\boldsymbol{T}), \boldsymbol{x}^{\prime}(0)=\boldsymbol{x}^{\prime}(\boldsymbol{T})$ has at least one $\boldsymbol{T}$-periodic solution on $\bar{\Omega}$.The following notations will be used throughout the rest of this study.

$$
\begin{aligned}
& |x|_{\infty}=\max _{t \in[0, T]}|x(t)|, \quad\left|x^{\prime}\right|_{\infty}=\max _{t \in[0, T]}\left|x^{\prime}(t)\right|, \quad|x|_{k}=\left(\int_{0}^{T}|x(t)|^{k} d t\right)^{\frac{1}{k}}, \\
& C_{T}^{1}:=\left\{x \in C^{1}(R, R): x(t+T)=x(T)\right\}, C_{T}:=\{x \in C(R, R): x(t+T)=x(T)\} . \\
& C_{T}, C_{T}^{1} \text { are two Banach spaces with the norms }\|x\|_{C_{T}^{1}}=\max \left\{|\boldsymbol{x}|_{\infty},\left|x^{\prime}\right|_{\infty}\right\},\|x\|_{C_{T}}=|x|_{\infty} .
\end{aligned}
$$

\section{The proof of theorem 1}

We are now in the position to give the proofs of Theorem 1.Proof. We will prove theorem in two steps.Existence.

We will show that Eq. (1) has at least one $\boldsymbol{T}$-periodic solution. Consider the homotopic equation of Eq. (1):

$$
\left(\phi\left(x^{\prime}(t)\right)\right)^{\prime}+\lambda C x^{\prime}(t)+\lambda \boldsymbol{g}(t, x(t))=\lambda \boldsymbol{e}(\boldsymbol{t}), \lambda \in(0,1),
$$

First, we prove that the set of the $\boldsymbol{T}$-periodic solution to Eq. (3) is bounded in $\boldsymbol{C}_{\boldsymbol{T}}^{1}$. Let $\boldsymbol{S} \subset \boldsymbol{C}_{\boldsymbol{T}}^{1}$ be the set of T-periodic solution of Eq. (3). If $\boldsymbol{S}=\Phi$, the proof is ended. Suppose $\boldsymbol{S} \neq \Phi$ and $\boldsymbol{x} \in \boldsymbol{S}$. Noticing that $x(0)=x(T), x^{\prime}(0)=x^{\prime}(T), \phi(0)=0$ and $\int_{0}^{T} e(t) d t=0$. Integrating from 0 to T of Eq. (3), we have $\int_{0}^{T} \boldsymbol{g}(\boldsymbol{t}, \boldsymbol{x}(\boldsymbol{t})) \boldsymbol{d} \boldsymbol{t}=0$, which implies that there exists a point $\boldsymbol{t}_{0} \in[0, \boldsymbol{T}]$ such that

$$
\boldsymbol{g}\left(\boldsymbol{t}_{0}, \boldsymbol{x}\left(\boldsymbol{t}_{0}\right)\right)=0 .
$$

By $\left(\mathrm{A}_{1}\right)$ and (4), we have $\left|\boldsymbol{x}\left(\boldsymbol{t}_{0}\right)\right|<\boldsymbol{d}$.

$$
\text { So }|\boldsymbol{x}(\boldsymbol{t})|<\boldsymbol{d}+\int_{0}^{T}\left|\boldsymbol{x}^{\prime}(s)\right| \boldsymbol{d s} \text {. That is }|\boldsymbol{x}|_{\infty}<\boldsymbol{d}+\left|\boldsymbol{x}^{\prime}\right|_{1} \text {. }
$$

In view of $\lim _{s \rightarrow+\infty} \alpha(s)=+\infty$, there is a constant $\boldsymbol{K}>0$ such that $\alpha(s) \geq 1, \forall \boldsymbol{s} \geq \boldsymbol{K}$. Define $\boldsymbol{E}_{1}=\left\{\boldsymbol{t}: \boldsymbol{t} \in[0,1],\left|\boldsymbol{x}^{\prime}(\boldsymbol{t})\right|>\boldsymbol{K}\right\}, \quad \boldsymbol{E}_{2}=\left\{\boldsymbol{t}: \boldsymbol{t} \in[0,1],\left|\boldsymbol{x}^{\prime}(\boldsymbol{t})\right|<\boldsymbol{K}\right\}$,

$\boldsymbol{E}_{3}=\{\boldsymbol{t}: \boldsymbol{t} \in[0,1],|\boldsymbol{x}(\boldsymbol{t})|>\boldsymbol{d}\}, \quad \boldsymbol{E}_{4}=\{\boldsymbol{t}: \boldsymbol{t} \in[0,1],|\boldsymbol{x}(\boldsymbol{t})|<\boldsymbol{d}\}$. By $\quad\left(\mathrm{H}_{2}\right)$ and (5), we obtain

$$
\int_{0}^{T}\left|x^{\prime}(t)\right|^{2} d t=\int_{E_{1}}\left|x^{\prime}(t)\right|^{2} d t+\int_{E_{2}}\left|x^{\prime}(t)\right|^{2} d t
$$

$$
\begin{aligned}
& \leq \int_{E_{1}}\left|x^{\prime}(t)\right|^{2} d t+K^{2} T \\
& \leq \int_{E_{1}} \frac{\phi\left(x^{\prime}(t)\right) \cdot x^{\prime}(t)}{\alpha\left(\left|x^{\prime}(t)\right|\right)} d t+K^{2} T \\
& \leq \int_{E_{1}} \phi\left(x^{\prime}(t)\right) x^{\prime}(t) d t+K^{2} T \\
& \leq \int_{0}^{T} \phi\left(x^{\prime}(t)\right) x^{\prime}(t) d t+K^{2} T \\
& =-\int_{0}^{T}\left(\phi\left(x^{\prime}(t)\right)\right)^{\prime} x(t) d t+K^{2} T \\
& =\lambda \int_{0}^{T} g(t, x(t)) \cdot x(t) d t-\lambda \int_{0}^{T} e(t) x(t) d t+K^{2} T
\end{aligned}
$$




$$
\begin{aligned}
& \lambda \int_{E_{3}} g(t, x(t)) \cdot x(t) d t+\lambda \int_{E_{4}} g(t, x(t)) \cdot x(t) d t \\
& -\lambda \int_{0}^{T} e(t) x(t) d t+K^{2} T \\
\leq & \lambda \int_{E_{4}} g(t, x(t)) \cdot x(t) d t-\lambda \int_{0}^{T} e(t) x(t) d t+K^{2} T \\
\leq & \int_{E_{4}}|g(t, x(t))| \cdot|x(t)| d t+\int_{0}^{T}|e(t) \| x(t)| d t+K^{2} T \\
\leq & \left(\max _{t \in[0,1],|x| \leq d}|g(t, x)|+|e|_{\infty}\right) T|x|_{\infty}+K^{2} T . \\
\text { Let } & M_{0}=\left(\max _{t \in[0,1],|x| \leq d}|g(t, x)|+|e|_{\infty}\right) T .
\end{aligned}
$$

Then we get

$$
\left|\boldsymbol{x}^{\prime}\right|_{2}^{2} \leq \boldsymbol{M}_{0}|\boldsymbol{x}|_{\infty}+\boldsymbol{K}^{2} \boldsymbol{T} \text {. }
$$

By (4), (6) and Holder inequality, we have

$$
\left|\boldsymbol{x}^{\prime}\right|_{1} \leq \sqrt{\boldsymbol{T}}\left(\boldsymbol{M}_{0}|\boldsymbol{x}|_{\infty}+\boldsymbol{K}^{2} \boldsymbol{T}\right)^{\frac{1}{2}} \leq \sqrt{\boldsymbol{T}}\left(\boldsymbol{M}_{0}\left(\boldsymbol{d}+\left|\boldsymbol{x}^{\prime}\right|_{1}\right)+\boldsymbol{K}^{2} \boldsymbol{T}\right)^{\frac{1}{2}}
$$

which yields that there exist a constant $\boldsymbol{M}_{1}>0$ such that

$\left|\boldsymbol{x}^{\prime}\right|_{1}<\boldsymbol{M}_{1},|\boldsymbol{x}|_{\infty}<\boldsymbol{d}+\boldsymbol{M}_{1}$.

Since $\boldsymbol{x}(0)=\boldsymbol{x}(\boldsymbol{T})$, there must exist $\boldsymbol{t}_{0}^{*}$ such that $\boldsymbol{x}^{\prime}\left(\boldsymbol{t}_{0}^{*}\right)=0$, from Eq. (1) we get

$$
\begin{aligned}
\left|\phi\left(x^{\prime}(t)\right)\right| & =\left|\int_{t_{0}^{*}}^{t}\left(\phi\left(x^{\prime}\right)\right)^{\prime} d s\right| \\
& =\lambda\left|\int_{t_{0}^{*}}^{t}\left(C x^{\prime}(s)+g(s, x(s))-e(s)\right) d s\right| \\
& \leq \int_{0}^{T}\left(C\left|x^{\prime}(s)\right|+\mid g(s, x(s)|+| e(s) \mid) d s\right. \\
& <C M_{1}+\left(G+|e|_{\infty}\right) T,
\end{aligned}
$$

where $\boldsymbol{G}=\max \left\{|\boldsymbol{g}(\boldsymbol{t}, \boldsymbol{x}): \boldsymbol{t} \in[0,1],| \boldsymbol{x} \mid \leq \boldsymbol{d}+\boldsymbol{M}_{1}\right\}$, so we have

$\left|\boldsymbol{x}^{\prime}\right|_{\infty}<\phi^{-1}\left(\boldsymbol{C M}_{1}+\left(\boldsymbol{G}+|\boldsymbol{e}|_{\infty}\right) \boldsymbol{T}\right)$.

Let $\boldsymbol{M}=\max \left\{\boldsymbol{d}+\boldsymbol{M}_{1}, \phi^{-1}\left(\boldsymbol{C} \boldsymbol{M}_{1}+\left(\boldsymbol{G}+|\boldsymbol{e}|_{\infty}\right) \boldsymbol{T}\right)\right\}$,

then from (7), (8),

$\|\boldsymbol{x}\|<\boldsymbol{M}$.

This means condition $\left(\mathrm{S}_{1}\right)$ of Lemma 1 is satisfied. Next we show condition $\left(\mathrm{S}_{2}\right),\left(\mathrm{S}_{3}\right)$ are also satisfied.

Set

$\tilde{f}\left(t, x(t), x^{\prime}(t)\right)=-C x^{\prime}(t)-g(t, x(t))+e(t)$,

thus

$\left(\phi\left(x^{\prime}(t)\right)\right)^{\prime}=\lambda \tilde{f}\left(t, x(t), x^{\prime}(t)\right)$

Denote $\mathrm{B}=\left\{\boldsymbol{x}: \boldsymbol{x} \in \boldsymbol{C}_{\boldsymbol{T}}^{1},\|\boldsymbol{x}\|<\boldsymbol{r}, \boldsymbol{r} \geq \boldsymbol{M}\right\}$, by $\quad\left(\mathrm{S}_{2}\right)$

$F(a)=\frac{1}{T} \int_{0}^{T} \tilde{f}(t, a, 0) d t=\frac{1}{T} \int_{0}^{T}(e(t)-g(t, a)) d t$

$=-\frac{1}{T} \int_{0}^{T} g(t, a) d t$, 
this together with $\left(\mathrm{A}_{1}\right)$, we get

$\boldsymbol{F}(\boldsymbol{r}) \boldsymbol{F}(-\boldsymbol{r})<0$

For $x \in \partial \Omega \cap \boldsymbol{R}, \mu \in[0,1]$,

$x \boldsymbol{H}(\boldsymbol{x}, \mu)=\mu \boldsymbol{x}^{2}-(1-\mu) \boldsymbol{x} \cdot \frac{1}{T} \int_{0}^{T} \boldsymbol{g}(\boldsymbol{t}, \boldsymbol{x}) \boldsymbol{d t}>0$,

we can see

$\operatorname{deg}(\boldsymbol{F}, \Omega \cap \boldsymbol{R}, 0\}=\operatorname{deg}(\boldsymbol{I}, \Omega \cap \boldsymbol{R}, 0) \neq 0$.

This means $\left(\mathrm{S}_{2}\right),\left(\mathrm{S}_{3}\right)$ of Lemma 1 hold. By applying Lemma 1 , there exists at least one solution with periodic $\boldsymbol{T}$ to Eq. (1). The existence is now completed.Uniqueness Let

$$
\begin{aligned}
& \psi(\boldsymbol{x})=\int_{0}^{x} \boldsymbol{C d u}=\boldsymbol{C x}, \boldsymbol{y}(\boldsymbol{t})=\phi\left(\boldsymbol{x}^{\prime}(\boldsymbol{t})\right)+\psi(\boldsymbol{x}(\boldsymbol{t})) . \\
& x^{\prime}(t)=\phi^{-1}[y(t)-\psi(x(t)], \\
& y^{\prime}(t)=-g(t, x(t))+e(t) \text {. } \\
& \text { Let } \boldsymbol{x}_{1}(\boldsymbol{t}) \text { and } \boldsymbol{x}_{2}(\boldsymbol{t}) \text { be two } \boldsymbol{T} \text {-periodic solution of Eq. (1), and } \\
& y_{i}(t)=\phi\left(x_{i}^{\prime}(t)\right)+\psi\left(x_{i}(t)\right), i=1,2 \text {. Then we obtain } \\
& x_{i}^{\prime}(t)=\phi^{-1}\left[y_{i}(t)-\psi\left(x_{i}(t)\right],\right. \\
& y_{i}^{\prime}(t)=-g\left(t, x_{i}(t)\right)+e(t) \text {. }
\end{aligned}
$$

Setting

$$
v(t)=x_{1}(t)-x_{2}(t), u(t)=y_{1}(t)-y_{2}(t),
$$

it follows from (10) that

$$
\begin{aligned}
& \boldsymbol{v}^{\prime}(\boldsymbol{t})=\phi^{-1}\left[\boldsymbol{y}_{1}(\boldsymbol{t})-\psi\left(\boldsymbol{x}_{1}(\boldsymbol{t})\right)\right]-\phi^{-1}\left[\boldsymbol{y}_{2}(\boldsymbol{t})-\psi\left(\boldsymbol{x}_{2}(\boldsymbol{t})\right)\right] \\
& \boldsymbol{u}^{\prime}(\boldsymbol{t})=-\left[\boldsymbol{g}\left(\boldsymbol{t}, \boldsymbol{x}_{1}(\boldsymbol{t})\right)-\boldsymbol{g}\left(\boldsymbol{t}, \boldsymbol{x}_{2}(\boldsymbol{t})\right)\right] .
\end{aligned}
$$

Now, we claim that $\boldsymbol{u}(\boldsymbol{t}) \leq 0, \forall \boldsymbol{t} \in \boldsymbol{R}$.

We argue by contradiction . Suppose there exists $\boldsymbol{t}_{2} \in(0, \boldsymbol{T})$ such that $u\left(\boldsymbol{t}_{2}\right)=\max _{t \in[0, \boldsymbol{T}]} \boldsymbol{u}(\boldsymbol{t})$, which implies that

$$
\begin{aligned}
& \boldsymbol{u}^{\prime}\left(\boldsymbol{t}_{2}\right)=-\left[\boldsymbol{g}\left(\boldsymbol{t}_{2}, \boldsymbol{x}_{1}\left(\boldsymbol{t}_{2}\right)\right)-\boldsymbol{g}\left(\boldsymbol{t}_{2}, \boldsymbol{x}_{2}\left(\boldsymbol{t}_{2}\right)\right)\right]=0 . \\
& \boldsymbol{u}^{\prime \prime}\left(\boldsymbol{t}_{2}\right)=-\left[\boldsymbol{g}_{1}{ }^{\prime}\left(\boldsymbol{t}_{2}, \boldsymbol{x}_{1}\left(\boldsymbol{t}_{2}\right)\right)+\boldsymbol{g}_{2}{ }^{\prime}\left(\boldsymbol{t}_{2}, \boldsymbol{x}_{1}\left(\boldsymbol{t}_{2}\right)\right) \boldsymbol{x}_{1}{ }^{\prime}\left(\boldsymbol{t}_{2}\right)\right. \\
& \left.-\boldsymbol{g}_{1}{ }^{\prime}\left(\boldsymbol{t}_{2}, \boldsymbol{x}_{2}\left(\boldsymbol{t}_{2}\right)\right)-\boldsymbol{g}_{2}{ }^{\prime}\left(\boldsymbol{t}_{2}, \boldsymbol{x}_{2}(\boldsymbol{t})\right) \boldsymbol{x}_{2}{ }^{\prime}\left(\boldsymbol{t}_{2}\right)\right] \leq 0, \\
& \text { where } \boldsymbol{g}_{1}{ }^{\prime}=\frac{\partial \boldsymbol{g}(\boldsymbol{t}, \boldsymbol{x})}{\partial \boldsymbol{t}}, \boldsymbol{g}_{2}{ }^{\prime}=\frac{\partial \boldsymbol{g}(\boldsymbol{t}, \boldsymbol{x})}{\partial \boldsymbol{x}} .
\end{aligned}
$$

By $\left(\mathrm{A}_{2}\right), \boldsymbol{g}_{2}^{\prime}(\boldsymbol{t}, \boldsymbol{x})<0$. So it follows from $\boldsymbol{g}\left(\boldsymbol{t}_{2}, \boldsymbol{x}_{1}\left(\boldsymbol{t}_{2}\right)\right)-\boldsymbol{g}\left(\boldsymbol{t}_{2}, \boldsymbol{x}_{2}\left(\boldsymbol{t}_{2}\right)\right)=0$ that $\boldsymbol{x}_{1}\left(\boldsymbol{t}_{2}\right)=\boldsymbol{x}_{2}\left(\boldsymbol{t}_{2}\right)$.

Thus, in view of $-\boldsymbol{g}_{2}{ }^{\prime}\left(\boldsymbol{t}_{2}, \boldsymbol{x}_{1}\left(\boldsymbol{t}_{2}\right)\right)>0, \boldsymbol{u}\left(\boldsymbol{t}_{2}\right)=\boldsymbol{y}_{1}\left(\boldsymbol{t}_{2}\right)-\boldsymbol{y}_{2}\left(\boldsymbol{t}_{2}\right)>0$,

Together with $\left(\mathrm{A}_{2}\right)$, we obtain $\boldsymbol{u}^{\prime \prime}\left(\boldsymbol{t}_{2}\right)=-\left[\boldsymbol{g}_{2}{ }^{\prime}\left(\boldsymbol{t}_{2}, \boldsymbol{x}_{1}\left(\boldsymbol{t}_{2}\right)\right) \boldsymbol{x}_{1}{ }^{\prime}\left(\boldsymbol{t}_{2}\right)-\boldsymbol{g}_{2}{ }^{\prime}\left(\boldsymbol{t}_{2}, \boldsymbol{x}_{2}(\boldsymbol{t})\right) \boldsymbol{x}_{2}{ }^{\prime}\left(\boldsymbol{t}_{2}\right)\right]$

$$
\begin{aligned}
& =-\boldsymbol{g}_{2}{ }^{\prime}\left(\boldsymbol{t}_{2}, \boldsymbol{x}_{1}\left(\boldsymbol{t}_{2}\right)\right)\left\{\phi ^ { - 1 } \left[\boldsymbol{y}_{1}\left(\boldsymbol{t}_{2}\right)-\right.\right. \\
& \left.\left.\psi\left(\boldsymbol{x}_{1}\left(\boldsymbol{t}_{2}\right)\right)\right]-\boldsymbol{\phi}^{-1}\left[\boldsymbol{y}_{2}\left(\boldsymbol{t}_{2}\right)-\psi\left(\boldsymbol{x}_{1}\left(\boldsymbol{t}_{2}\right)\right)\right]\right\}>0,
\end{aligned}
$$

which contradicts with $\boldsymbol{u}^{\prime \prime}(\boldsymbol{t}) \leq 0$. Now, we have proved that $\boldsymbol{u}(\boldsymbol{t}) \leq 0, \forall \boldsymbol{t} \in \boldsymbol{R}$.

Analogously, one can show that $\boldsymbol{u}(\boldsymbol{t}) \geq 0, \forall \boldsymbol{t} \in \boldsymbol{R}$.

So we have $u(t) \equiv 0$. That is $x_{1}(t)=x_{2}(t), \forall t \in R$

Hence, Eq. (1) has an unique $\boldsymbol{T}$-periodic solution. The proof of Theorem 1 is now completed 


\section{References}

[1] X.Q. Liu, Y.X. Guo and J.Q. Liu, Solutions for singular p-Laplacian equation in $\boldsymbol{R}^{N}$, Jrl Syst Sci \& Complexity (2009) 22: 597-613.

[2] S.P. Lu, Z.J. Gui, On the existence of periodic solutions to p-Laplacian Rayleigh differential equation with a delay, J. Math. Anal. Appl. 325(2007)685-702.

[3] W.S. Cheung, J.L. Ren, Periodic solutions for p-Laplacian Liénard equation with a deviating argument, Nonlinear Anal. 59(2004)107-120.

[4] S.P. Lu, W.G. Ge, Periodic solutions for second order p-Laplacian differential equation with a deviating argument (in Chinese), Acta. Math. Sin. (2005) 48 (5)841-850.

[5] S.P. Lu, W.G. Ge, Periodic solutions for a kind of Liénard equations with deviating arguments. J. Math. Anal. Appl. 249 (2004)231-243. 